
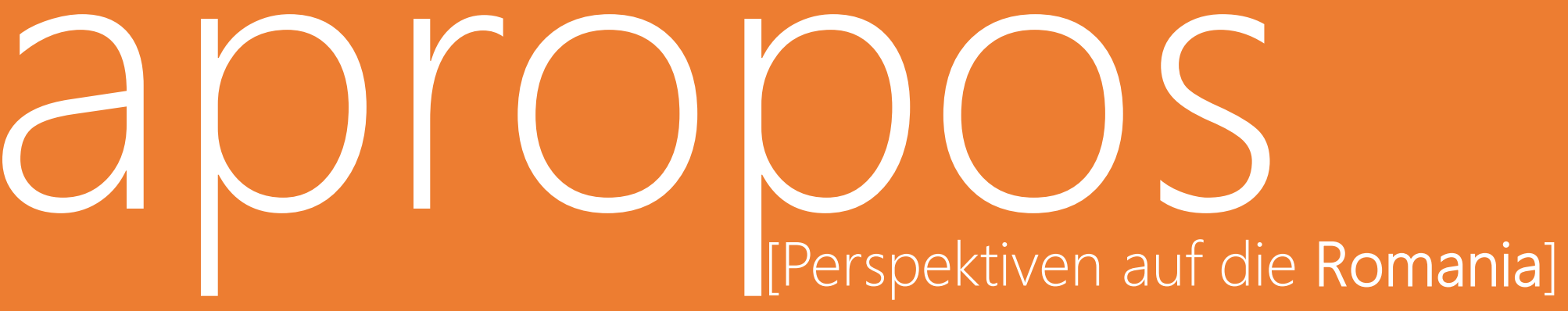

Sprache/Literatur/Kultur/Geschichte/Ideen/Politik/Gesellschaft

Rezension von DUfTER, Andreas, Klaus Grübl \& Thomas Scharinger (ed.). 2020. Des parlers d'oil à la francophonie. Contact, variation et changement linguistiques. Berlin/Boston: de Gruyter

Roger Schöntag

apropos [Perspektiven auf die Romania] hosted by Hamburg University Press

2021, 6

pp. 280-287

ISSN: 2627-3446

Online

https://journals.sub.uni-hamburg.de/apropos/article/view/1721

Zitierweise

Schöntag, Roger, 2021. „Rezension von DUFTER, Andreas, Klaus Grübl \& Thomas Scharinger (ed.). 2020. Des parlers d'oill à la francophonie. Contact, variation et changement linguistiques. Berlin/Boston: de Gruyter", apropos [Perspektiven auf die Romania] 6/2021, 280-287. doi: 10.15460/apropos.6.1721 
Roger Schöntag

Rezension

\title{
DUFTER, Andreas, Klaus Grübl \& Thomas Scharinger (ed.). 2020. Des parlers d'oïl à la francophonie. Contact, variation et changement linguistiques. Berlin/Boston: de Gruyter.
}

\author{
Roger Schöntag \\ ist akademischer Rat (a.Z.) am Institut \\ für Romanistik (Sprachwissenschaft) \\ der Universität Erlangen. \\ roger.schoentag@fau.de
}

Keywords

Französische Sprachgeschichte - Frankophonie - Sprachwandel - Sprachkontakt Standardisierung

Der vorliegende Sammelband ist das Ergebnis der Tagungsreihe Repenser l'histoire du français, die in ihrer letzten Ausgabe vom 7.-8. April 2016 in München stattfand und die einst von Maria Iliescu (1927-2020) und David Andrew Trotter (1957-2015) in Innsbruck begründet wurde. ${ }^{1}$ Der im Titel zusammengefasste Schwerpunkt des Bandes, nämlich historische Sprachwandelprozesse im Französischen unter besonderer Berücksichtigung der Varietäten vom Mittelalter bis zur Ausbreitung des Französischen im Zuge der kolonialen Expansion, wird nochmals durch drei Sektionen präzisiert: 1) Contact, nivellement et (re-)standardisation : De la variation médieval au français moderne, 2) Expansion du français comme langue seconde ou véhiculaire und 3) Continuités et ruptures en français d'Outre-mer et dans l'émergence des langues créoles.

Nach einigen grundsätzlichen Überlegungen der Herausgeber beschäftigt sich Zinaïda Geylikman im ersten Beitrag mit einer bekannten morphologischen Alternanz im Altfranzösischen: "Tu parli como ber": Le destin de la forme monosyllabique du substantif ,baron' dans la "Geste francor". Bedingt durch die Zweikasusdeklination gibt es im Altfranzösischen eine mono- und entsprechende bisyllabische Form zur Bezeichnung eines Edelmanns, und zwar ber (Rektus Singular) und baron (Obliquus Singular) sowie baron (Rektus Plural) und barons

\footnotetext{
${ }^{1}$ Zu den bisherigen Publikationen, die aus dieser Tagungsreihe hervorgegangen sind, zählen Lagorgette (2014) und Kristol (2017). Zu weiteren Kongressreihen sowie dem Stand der historischen Sprachforschung zum Französischen allgemein cf. Schöntag (2018).
} 
(Obliquus Plural), wobei allein die Obliquus-Formen (baron/barons) bis ins Neufranzösische erhalten bleiben. Um den Veränderungen im Gebrauch der beiden Formen auf die Spur zu kommen, untersucht Geylikman die Verwendung von ber und baron in der Geste francor (1. Hälfte 14. Jh.), einer franko-italienischen Sammlung von chansons de geste. Zunächst werden jedoch die verschiedenen Funktionen im altfranzösischen Heldenepos näher beleuchtet. Dabei ist festzustellen, dass beide Formen, ber und baron, als épithète formulaire auftreten können, mit jedoch eindeutiger Präferenz für das monosyllabische ber (55:4 Okkurenzen im 12. Jh.; 73:7 im 13. Jh.): z. B. Rollant i fut e Oliver li ber, A tant $i$ vint Willame le barun (p. 23). Beim Gebrauch als Prädikativ überwiegt ebenfalls die monosyllabische Form, nicht selten begleitet von dem Intensivierungsadverb mult: z. B. Li quens Guillelmes fut molt gentis et ber (p. 25). Die zentrale These Geylikmans lautet darauf aufbauend, dass sich schon im 12. Jh. eine funktionale und semantische Differenzierung anbahnt, die sich durch den adjektivischen Gebrauch von vorwiegend ber mit valeur évaluative andeutet, wobei jedoch zunächst auch noch baron diese Funktion übernehmen kann, da beide noch zum gleichen Paradigma gehören; eine deutliche Differenzierung setzt erst später ein. Im Mittelfranzösischen zeigt sich dann adjektivisch eine Verwendung von ber, die nicht mehr an die Grundbedeutung, valeureux' (in Bezug auf einen homme aristocrate) gebunden ist, sondern metonymisch davon abgleitet wurde und sich auf Sachen beziehen kann: z. B. La response dou duc de Bourgogne au cuer ber (p. 28). In der Analyse der Geste francor zeigt sich schließlich ein unabhängiger Gebrauch von ber und baron, da der Zusammenhang der beiden Formen im Zweikasussystem des Altfranzösischen hier nicht mehr gesehen wurde. Für die italophonen Schreiber war die Form ber opak und wurde, wenn überhaupt, als eigenständiges Adjektiv wahrgenommen, und zwar mit einer allgemeinen Bedeutung, de valeur', applizierbar auf Personen im Allgemeinen, die nicht unbedingt aristokratisch sein mussten (p. 4142). Der Beitrag von Geylikman liefert einen wertvollen Einblick in einen komplexen Sprachwandelprozess vom Alt- zum Mittelfranzösischen, den man sicherlich mit Gewinn noch in weiteren Studien vertiefen kann, im Hinblick auf die Quellenbasis und auch auf ähnliche Formen ohne Stammausgleich (z. B. afrz. copain vs. compagnon; cuens vs. conte; lédre/lérre vs. larron).

Der folgende Beitrag von Annie Bertin betrifft einige Réflexions sur un remaniement picardisant de l' "Histoire d'Alexandre" à la fin du $15^{e}$ siècle. Hierbei wird die französische Übersetzung der Alexandergeschichte des Portugiesen Vasque de Lucène (1435-1512) in den Fokus genommen, der die lateinische Vorlage des römischen Historikers Quintus Curtius Rufus (1. Jh. n. Chr.), die Historiae Alexandri Magni Macedonis, bearbeitet hat. Das Werk des portugiesischen Gelehrten, der in Coimbra, Paris und Köln studiert hatte und im Dienste Isabellas von Portugal (13971471), der dritten Ehefrau des Herzogs Phillip II. des Guten von Burgund (13961467, Hzg. ab 1419), stand, ist in zwei Manuskriptversionen überliefert. Dabei wird deutlich, wie der Text der Histoire d'Alexandre $(1468)^{2}$ in einer Überarbeitung (2.

\footnotetext{
2 Der vollständige Titel lautete Les faits et gestes d'Alexandre le grant compilez de plusieurs livres et adjoints aus histoires de Quinte Curce Rufe, was auch damit zusammenhängt, dass Vasque de Lucène die im Original
} 
Manuskript) entgegen der allgemeinen Tendenzen der Entpikadisierung sich der lokalen scripta Flanderns und der Pikardie annähert und sich ein Stück weit dem françois bzw. der Franzisierung verweigert. Die Annäherung an eine maniere de parler des einfachen Volkes impliziert zudem eine Delatinisierung, vor allem der Syntax. Dieser hier aufgezeigte Prozess, der deutlich macht, dass die Standardisierung des Französischen (bzw. Franzischen) nicht linear verlief, ist mehr als beachtenswert.

In ihrem Beitrag Les locutions conjonctives 'malgré que' et 'à cause que' : Normes et usages en diachronie geht Julie Glikman der Entstehungsgeschichte dieser heute durchaus verbreiteten, aber als fehlerhaft im Sinne der präskriptiven Norm angesehenen Konjunktionen auf den Grund. Interessant ist dabei nicht nur die unterschiedliche historische Genese - à cause que (16./17. Jh.), malgré que (18. Jh.) -, sondern auch die zum Schluss erhobene Sprecher-Akzeptabilität der beiden ehemals unmarkierten und heute als familier bzw. populaire eingestuften Lokutionen.

In Contextes promoteurs et émergence des questions 'in situ' en français untersucht Pierre Larrivée einen bestimmten Typus von Fragesatz (z. B. Paul a parlé à qui?), der erstmals im 15. Jh. auftretend vor allem in Texten mit Passagen fingierter Mündlichkeit ab dem 18. Jh. erscheint, oftmals parallel mit einem anderen Typus, der als interrogation retardée (z. B. Paul en a parlé; à qui?) bezeichnet wird.

Die zweite Sektion (Expansion du français comme langue seconde ou véhiculaire) beginnt mit dem Artikel von Andres Kristol zum Thema François Poulain de la Barre et les "Remarques particulières sur la Langue Françoise pour la ville de Genève" (1691). Les enseignements de la première cacologie connue d'un français régional, in dem ein bisher wenig beachteter normativer Text im Gefolge von Claude Favre de Vaugelas' (1585-1650) Remarques sur la Langue Françoise (1647) untersucht wird. François Poul(I)ain de la Barre (1647-1723), der vor allem als kartesianischer Philosoph mit Publikationen zur querelle des femmes, in denen er progressive Ansichten zur éducation féminine vertritt, bekannt geworden ist (cf. De l'Égalité des deux sexes, 1673; De l'Éducation des dames, 1674; De l'Excellence des hommes contre l'égalité des sexes, 1675), hat sich auch mit sprachlichen Problemen auseinandergesetzt. Neben einer Schrift zu Übersetzungsproblemen (cf. Les Rapports de la langue latine avec la françoise pour traduire élégament et sans peine, 1672) widmet er ein Traktat den sprachlichen Charakteristika seiner neuen Heimatstadt Genf, in die er nach seiner Konversion zum Protestantismus übersiedelte (1688) und wo er zeitweilig als Sprachlehrer arbeitete. Zu dieser Zeit sind in der Suisse romande noch das Frankoprovenzalische bzw. seine Varietäten stark präsent und das Französische ist auf eine gehobene Bürgerschicht begrenzt. In seinem Essai des Remarques particulières sur la Langue Françoise pour la ville de Genève (1691) versucht Poulain nun, seinen Mitbürgern ein bon français bzw. den bon usage näherzubringen, in dem er unter verschiedenen Rubriken die seiner Meinung nach

fehlenden Bücher bzw. Passagen der ursprünglichen 10 Bücher der Alexandergeschichte des Curtius Rufus ergänzte (es fehlen die Bücher 1-2, der Beginn des 3., das Ende des 5, der Beginn des 6. und Teile des 10., was jedoch zumindest partiell aus Diodor, Justin, Plutarch und Arrian rekonstruiert werden kann). 
zugrundeliegenden Normverstöße auflistet: I. Des mots qui sont hors d'Usage (z. B. débonnaire, moleste, prou), II. Des Mots Impropres (z. B. censurer, tirant, y statt le/lui/cela: cf. I'y verrai statt je le verrai), III. Un Genre pour un autre (z. B. un afaire, un huile), IV. Un Mode pour un autre (z. B. passé surcomposé: cf. I'ay eu dit cela) (p. 124-136). Unter diesen Kategorien stigmatisiert er, nicht selten unter Rückgriff auf das Dictionnaire françois contenant les mots et les choses (1680) von César-Pierre Richelet (1626-1698), bestimmte Auffälligkeiten des Französischen von Genf. In seiner Analyse einzelner Lexeme und Konstruktionen präpariert Kristol mit großer Präzision heraus, wie sich die Genese dieses schweizerischen Französischen vollzieht und durch die Adaption von Frankprovenzalismen, Dialektismen, Archaismen und auch Innovationen ein eigener Regiolekt entsteht.

Im anschließenden Beitrag von Joachim Steffen L'entrée dans l'écrit revisitée: Éléments occitans dans les lettres de soldats peu-lettrés du Langudedoc-Rousillon (Révolution et Empire) geht es um den Einfluss des Okzitanischen bei ungebildeten Schreibern des Französischen, also solchen, die zwar alphabetisiert sind, deren Texte jedoch orthographische, lexikalische, morphosyntaktische und diskurstraditionelle Normverstöße aufweisen (cf. frz. peu-lettrés, it. semicolti). Die Auswertung der Soldatenbriefe zu Beginn des 19. Jhs. erfolgt anhand eines Teils des umfangreichen Corpus Historique du Substandard Français (CHSF), initiiert von Harald Thun (cf. z. B. Thun 2018). ${ }^{3}$ Basis der Untersuchung sind 60 Briefe aus den Archives départementales de l'Hérault (Teil des CHSF) von Soldaten der Revolutionsarmee (datiert 1793-1795), die aus der Gemeinde Lodève nördlich von Montpellier stammen. Diese Region war bis zu Beginn des 20. Jhs. im Wesentlichen okzitanischsprachig, d. h. es bestand eine Diglossie mit dem Französischen, welches ab dem Edikt von Villers-Cotterêts (1539) zunehmend als Schriftsprache und auch als high variety in der Mündlichkeit fungierte. Bemerkenswert an diesen Briefen ist, dass sie ja vor der Zeit der großen Schulpflichtoffensive des 19. Jhs. entstanden (cf. loi Guizot, 1833; loi Ferry 1882), in einer Epoche, die bezüglich der Schreibfähigkeit der couches populaires noch kaum untersucht ist. Wie Steffen zu Recht kritisiert lässt sich die Schreib- und Lesefähigkeit nur sehr approximativ an der Signaturfähigkeit ablesen, ein vielleicht etwas besserer - wenn auch nicht verlässlicher Indikator scheint die Verbreitung bzw. Auflage der bibliothèque bleue sowie die Existenz von ca. 30.000 petites écoles unter kirchlicher Aufsicht. ${ }^{4}$ Die in den Briefen

\footnotetext{
${ }^{3}$ Neben dem CHSF - Corpus Historique du Substandard Français von Harald Thun (Kiel), welches einen größeren Zeitraum (1789-1918) umfasst, sei auf weitere Datenbank-Korpora mit Texten der peu-lettrés hingewiesen, und zwar das Corpus14, geleitet u. a. von Nathalie Auger (Montpellier) und Sonia Branca-Rosoff (Sorbonne), welches Briefe der Soldaten (poilus) des 1. Weltkrieges (1914-1918) beinhaltet, auf das angeschlossene Projekt Egoling14-18 von Sybille Große (Heidelberg) zur gleichen Epoche sowie auf das CDPB - Corpus des bagnards et leurs proches von Stephanie Massicot (Erlangen), in dem Briefe von Strafgefangenen (bagnards) und ihren Angehörigen (1854-1892) aufbereitet wurden. Ein Korpus, das sich einer älteren Sprachstufe verschrieben hat, ist das von Pierre Larrivée (Caen) geleitete Projekt EPELE - L'écriture des peu-lettrés zum Französischen der Normandie (14.-16. Jh.). Rezente Studien dazu sind u. a. Steffen, Thun \& Zaiser (2018), Große \& Sowada (2020) sowie Massicot (2020).

${ }^{4}$ Mit bibliothèque bleue oder Livres bleues bezeichnet man Bücher populärer Unterhaltungsliteratur (oft Prosafassungen altfranzösischer Versromane), die ab dem Beginn des 17. Jhs. weite Verbreitung fanden (cf. z. B. aus der Druckerei der frères Oudot in Troyes) und die einen einfachen blauen Umschlag hatten (cf. dt. Volksbücher, Kolportageromane).
} 
der einfachen Soldaten festgestellten Devianzen, also Abweichungen von der Norm, betreffen die verschiedensten Bereiche des Sprachlichen. Dabei gelingt es Steffen u. a. folgende auf okzitanischen Einfluss zurückzuführenden Merkmale zu isolieren: 1) Aufgrund der Entphonologisierung von /b/ und / $/$ im Languedokischen (und Gaskognischen) ergeben sich Schreibungen wie au cam de vatalle ('au champ de bataille') oder le oubrage ('I'ou rage') (p. 152-153); 2) aufgrund des gerollten $r$ im Okzitanischen, ergeben sich Schreibungen mit den artikulatorisch nahestehenden Lauten /d/ oder /// wie in éjéné vous é cride jamais ('Et je ne vous écrirai jamais') oder A Escalene ('À Escarene') (p. 153-154); 3) die Bildung des passé composé mit avoir statt mit être wie im Okzitanischen, z. B. nous avons rete ('nous sommes restés') oder il a parti ('il estparti') (p. 158). Ein zweifellos wichtiger Beitrag zur weiteren Erforschung der écriture populaire.

Der folgende Artikel von Joseph Reisdoerfer, Histoire linguistique des français: Éléments pour une histoire du français au grand-duché de Luxembourg zeichnet die Geschichte des Französischen im Großherzogtum Luxemburg seit dem 12. Jh. nach, in welchem es bis heute als offizielle Amts- und Schulsprache fungiert, obwohl es aufgrund territorialer Einbußen schon lange keine autochthonen native speakers mehr gibt.

Im Anschluss beschäftigt sich Clémentine Rubio mit der Diffusion du français en Palestine ottomane et idéologies linguistiques; dabei handelt es sich um ein Kapitel der Geschichte der Frankophonie in einem nicht von Frankreich kolonisierten Landstrich, in dem das Französische jedoch als imperiale Sprache mit zivilisatorischen Ansprüchen wirkte (cf. français de coeur vs. français de race).

Hervorzuheben ist auch die bisher noch kaum beachtete Stellung des Französischen in Finnland, welche von Juhani Härmä in seinem Beitrag Le français et le suédois dans les correspondances finlandaises des $18^{e}$ et $19^{e}$ siècles : Contacts de langues bearbeitet wurde. ${ }^{5}$ Härmä sichtet die Korrespondenzen zweier Gelehrter im finnischen Nationalarchiv (fin. Kansallisarkisto, schw. Riksarkivet), und zwar von dem architektonischen Planer Helsinkis Johan Albrecht Ehrenström (1762-1867) und von dem Diplomaten und Höfling Gustaf Mauritz Armfelt (1757-1814). Finnland gehörte über viele Jahrhunderte zum schwedischen Königreich (12.-18. Jh.), bis es 1809 von Russland annektiert wurde. Neben dem Finnischen als einheimische Sprache waren im 17./18. Jh. das Schwedische, das Russische, das Deutsche, das Lateinische und das Französische in Gebrauch. Dabei fungierte das Französische wie zu dieser Zeit auch in anderen Teilen Europas als lingua franca und Sprache der Oberschicht, zumal die gegenseitige Verständigung der anderen Sprachgruppen eingeschränkt war (Finnen, Schweden, Russen). So findet man in den untersuchten Korrepondenzen verschiedene französische Entlehnungen unterschiedlicher Integration bis hin zu Code-Switching: z. B. „och hvilken således måste fullbordas, en depit af alla dumma anmärkningar och critiques, hvilka uphöra så snart det Herculanska arbetet står färdigt för deras ögon. Då heter det: qui

\footnotetext{
${ }^{5}$ In der von den Herausgebern vorangestellten Zusammenfassung ist bezüglich dieses bzw. des vorherigen Artikels die Reihenfolge vertauscht worden (p. 8) - une faute de négligence.
} 
I'auroit crû?" (p. 219). Eine weitere Auswertung der Quellen und Systematisierung der sprachlichen Phänomene ist hierbei zweifellos lohnenswert.

Die dritte Sektion (Continuités et ruptures en français d'Outre-mer et dans l'émergence des langues créoles) beginnt mit einem Beitrag von Ingrid NeumannHolzschuh und Julia Mitko zum Französischen in Nord-Amerika: "Tout le monde parle différent mais on se comprend pareil": Le rôle de l'adjectif-adverbe dans le français nord-américain. Basierend auf verschiedenen Studien von Hummel (cf. z. B. Hummel 2017) wird der mündliche Sprachgebrauch des kanadischen Französisch (cf. z. B. CFPQ - Corpus de français parlé au Québec) sowie des français louisianais hinsichtlich der Verwendung von bestimmten Adverbtypen untersucht. Dabei wird deutlich, dass sich die im heutigen Standardfranzösisch dominierende Form des Typs -ment, die sich erst mit der Scholarisiserung im 19. Jh. durchzusetzen beginnt, in den nordamerikanischen Varietäten (cf. québécois, acadien, louisianais etc.) zumindest in der älteren Bevölkerung deutlich marginaler auftritt, zugunsten von Alternativkonstruktionen wie dem adjektivisch gebrauchten Adverb (cf. z. B. II a mouillé terrible, Ça se comprend réel bon, je vas sûr le faire) (p. 249, 251, 252). ${ }^{6}$ Ein wichtiges Indiz für die Sprachentwicklung jenseits der Norm beiderseits des Atlantiks.

France Martineau und Wim Reymsen behandeln in Bouleversements sociaux et normes orthographiques: L'exemple du Régime anglais dans l'histoire du français québécois graphische Auffälligkeiten in den Korrespondenzen der Familie Papineau ${ }^{7}$ über zwei Generationen hinweg bezüglich der Merkmale ere/ére/ère (z. B. mère), es/oi/ai (z. B. françois) sowie ans/ens bzw. ants/ents (z. B. enfants). Hieraus lässt sich, zumindest in Ansätzen, herauslesen, inwieweit orthographische Innovationen aus Frankreich im englisch besetzten frankophonen Kanada (cf. Régime anglais 1760-1867) Fuß fassen können; sekundär sind dadurch auch Rückschlüsse auf das gesellschaftliche Netzwerk der Oberschicht und den sprachlichen Einfluss einzelner Protagonisten möglich.

Im letzten Artikel des Bandes Dialectes - créolisation - convergence: Quelques hypothèses à partir du berrichon et du poitevin-saintongeais versuchen Sibylle Kriegel, Ralph Ludwig und Stefan Pfänder mit Hilfe eines typologischen Vergleiches dialektaler Charakteristika (z. B. J'avons) Parallelen zu ähnlichen Phänomenen in französisch basierten Kreols (cf. créole haïtien, créole guadeloupéen, créole seychellois) zu ziehen. So interessant und aufschlussreich die Einzelanalyse sein mag, die sicherlich Grundprinzipien der Kreolisierung näher beleuchtet, so bleibt eine gewisse Skepsis, wenn die Entwicklungen aus ihrem historischen Kontext gerissen werden. ${ }^{8}$ So entspricht beispielsweise der Verlust des Negationspartikels

\footnotetext{
${ }^{6}$ Interessant sind auch die auf englische Interferenzen zurückgehende Formen wie wellment (wayment) oder full (z. B. full spécial) (p. 252). Die Formen mit -ment dienen häufig der Intensivierung (z. B. joliment, tellement) oder der Fokalisierung (z. B. seulement).

${ }^{7}$ Cf. Joseph Papineau (1752-1841), Louis-Joseph Papineau (1786-1871), Denis-Benjamin Papineau (1789-1854).

${ }^{8}$ Etwas unglücklich gewählt sind zudem die Beispiele, die die Vorteile des Begriffs der Kopie illustrieren sollen, denn engl. weekend > frz. week-end (cf. copiage ouverte) vs. engl. skyscraper > frz. gratte-ciel (cf. copiage couverte) entspricht exactement der Unterscheidung äußeres Lehngut vs. inneres Lehngut im traditionellen Betz'schen Lehngut-Schema (cf. Betz 1949: 27-28), das zudem weitere Differenzierungen bietet. Wenn also
} 
ne im berrichon (z. B. J'v'lons pas nous en torner) eben nicht der Verwendung von pa im créole guadeloupéen (z. B. An pa chonjé sa), was sich allein daran zeigt, dass pa im Kreol präverbal auftritt, $d$. h. eine ganz andere Genese hat als der Wechsel von afrz. ne über ne...pas zu pas. Unabhängig von der Kontinuitätsfrage in der Kreolistik (cf. rupture vs. continuité) scheinen solche Phänomene doch für eine bestimmte Form der Reanalyse zu sprechen, die sich in den französischen Dialekten so nicht findet. Der Ansatz, auf dialektale Erscheinungen zum besseren Verständnis der Entstehung von Phänomenen in den Kreolsprachen zurückzugreifen, bleibt, davon unberührt, sicherlich interessant und fruchtbar.

Insgesamt bietet der sehr sorgsam gestaltete Sammelband einen wertvollen Einblick in verschiedene Einzelentwicklungen des Französischen, wobei - und das ist durchaus hervorzuheben - die (diatopischen) Varietäten eine maßgebliche Rolle einnehmen. Gerade auch die Bandbreite der behandelten Grenzbereiche der frankophonen Sprachgeschichte (Französisch in Luxemburg, Palästina und Finnland, Kreolsprachen, peu-lettrés) ist bemerkenswert. ${ }^{9}$

So fügt sich dieser Kongressband von Dufter et al. in andere in letzter Zeit erschienene, wichtige Publikationen zum Sprachwandel ein (cf. z. B. Ayres-Bennett et al. 2018) und leistet einen profunden Beitrag zur französischen Sprachgeschichte.

\section{Bibliografie}

Ayres-BenNetT, Wendy et al. (ed.). 2018. Nouvelles voies d'accès au changement linguistique, Paris: Classiques Garnier.

BETZ, Werner. 1949. Deutsch und Lateinisch. Die Lehnbildungen der althochdeutschen Benediktinerregel. Bonn: Bouvier.

GROBE, Sybille \& Lena Sowada. 2020. "Socialisation écrite et rédaction épistolaire de scripteurs moins expérimentés : lettres des soldats de la Grande Guerre." Romanistisches Jahrbuch 71, 82-129.

HuMmeL, Martin. 2017. „Adjectives with adverbial functions in Romance." In Adjective adverb interfaces in Romance, ed. Hummel, Martin \& Valera Salvador, 13-46, Amsterdam \& Philadelphia: Benjamins.

KRISTOL, Andreas (ed.). 2017. La mise à l'écrit et ses conséquences. Actes du troisième colloque "Repenser I'histoire du français", Université de Neuchâtel, 5-6 juin 2014, Tübingen: Francke.

LAgORGETTE, Dominique (ed.). 2014. Repenser I'histoire du français, Chambéry: Université de Savoie.

MASSICOT, Stephanie. 2020. Versprachlichungsstrategien unroutinierter Schreiber: französische Bittbriefe des 19. Jahrhunderts auf dem Prüfstand.

\footnotetext{
Argumente für die Verwendung von copy statt borrowing sprechen, müssten diese auf einer anderen Ebene liegen. Zu einer Übersicht verschiedener Entlehnungsmodelle in Erweiterung der Betz'schen Kategorien cf. Schöntag (2009: 56-72).

9 Trotz der präsentierten Sprachenvielfalt und der Internationalität der Kongressteilnehmer sind alle Beiträge auf Französisch verfasst. Rein formal versteht man die Herausgeber, dass hier Objektsprache und Metasprache homogen gehalten wurden, dennoch ist es auch bedauerlich, denn Mehrsprachigkeit hat in der Romanistik durchaus Tradition und so mutet es ein wenig befremdlich an, dass die Publikation einer Münchner Tagung, die auch noch in der renommierten Reihe Beihefte zur Zeitschrift für romanische Philologie erscheint, nicht an die polyglotte Tradition anknüpft.
} 
Erlangen: Friedrich-Alexander-Universität Erlangen-Nürnberg [Dissertation]. <https://opus4.kobv.de/opus4-fau/frontdoor/index/index/docld/15658>, 22.02.2021.

SCHÖNTAG, Roger. 2009. Sprachkontakt: Grammatische Interferenz im Französischen? Der Einfluß des Englischen auf das Stellungsverhalten des attributiven Adjektivs/Contact de langues: Interférence grammaticale en français? L'influence anglaise sur la position de l'adjectif épithète. München: Utz.

SCHÖNTAG, Roger. 2018. „Aktueller Forschungsstand: Französische Sprachgeschichte." In Seitenblicke auf die französische Sprachgeschichte. Akten der Tagung Französische Sprachgeschichte vom 13.-16. Oktober 2016 an der Ludwig-Maximilians-Universität München. Sektionen: Interne Sprachgeschichte, Sprachwissenschaftsgeschichte, Kreolsprachen, Okzitanisch, Semicolti/Peu-lettrés, Französisch außerhalb Frankreichs - Sprachkontakt, ed. Schäfer-Prieß, Barbara \& Roger Schöntag, 19-36, Tübingen: Narr.

SteFFEN, Joachim, Harald Thun \& Rainer Zaiser (ed.). 2018. Classes populaires, scripturalité et histoire de la langue. Un bilan interdisciplinaire. Kiel: Westensee.

Thun, Harald. 2018. „Substandard und Regionalsprachen. Das Corpus Historique du Substandard Français, die écriture populaire und die écriture alternative (1789-1918)." In Seitenblicke auf die französische Sprachgeschichte. Akten der Tagung Französische Sprachgeschichte vom 13.-16. Oktober 2016 an der Ludwig-Maximilians-Universität München. Sektionen: Interne Sprachgeschichte, Sprachwissenschaftsgeschichte, Kreolsprachen, Okzitanisch, Semicolti/Peu-lettrés, Französisch außerhalb Frankreichs - Sprachkontakt, ed. Schäfer-Prieß, Barbara \& Roger Schöntag, 257-303, Tübingen: Narr.

\section{Korpora}

Auger, Nathalie et al. (ed.). 2013ff. Corpus 14. Montpellier: Laboratoire PRAXILING (UMR 5267, Université Paul-Valéry Montpellier-CNRS). $<$ https://www.univ-montp3.fr/corpus14/>, 22.01.2021.

GroßE, Sybille (ed.). 2019. Egoling14-18. Heidelberg: Universität Heidelberg. $<$ https://heidelberger-forum-edition.de/egoling14-18>, 22.02.2021.

LARIVEE, Pierre (ed.). 2018. EPELE - L'écriture des peu-lettrés. Caen: Université de Caen Normandie. <https://www.unicaen.fr/epele//accueil>, 22.02.2021.

MASSICOT, Stephanie (ed.). 2018. CDPB - Corpus des bagnards et leurs proches. Erlangen: Friedrich-Alexander-Universität Erlangen-Nürnberg (FAU). <http://geuselambix.phil.uni-erlangen.de:3000/>, 22.02.2021.

THuN, Harald (ed.). CHSF - Corpus Historique du Substandard Français. Kiel: Christian-Albrechts-Universität zu Kiel (CAU). $<$ https://www.romanistik.uni-kiel.de/de/personen-und-sprechzeiten/THUN/introduction>, 22.02.2021. 